\title{
Sinus Arrest by ECG Finding
}

National Cancer Institute

\section{Source}

National Cancer Institute. Sinus Arrest by ECG Finding. NCI Thesaurus. Code C62242.

An electrocardiog raphic finding in which a failure of impulse formation or conduction in the sinus node produces prolong ation of the P-P interval or dropped P waves. The threshold for the prolong ation of the P-P interval is not well defined. (CDISC) 